\title{
THE FITTING OF LINEAR REGRESSION LINES BY THE METHOD OF LEAST SQUARES
}

\author{
By T. W. Dwight, \\ University of Toronto
}

T IS the purpase of this article to outline the basis of the algebraic method of fitting linear regression lines in a somewhat different way from that followed by most elementary text books on statistical methods, with the idea of bringing out as clearly as possible just why the algebraic calculations involved give the equation of a line that is related to the basic data in the best manner possible. Some space will be devoted to the questions of symbols and to methods of tabulation and computation of data. A lack of a clear understanding of these points is often a major diffculty to anyone encountering statistical methods for the first time. The symbols used are taken mainly from Mills*.

The algebraic method of fitting a straight regression line involves the determination of the equation defining the position of the line. This is usually written in statistical work in the form

$$
\mathrm{Y}_{\mathrm{c}}=\mathrm{a}+\mathrm{bX}
$$

where $Y_{c}$ is any value corresponding to a point in the line, a the value of $\mathrm{Y}_{\mathrm{c}}$ when $\mathrm{X}=0$, and $\mathrm{b}$ the slope of the line. Mathematically, the problem is to find suitable numerical values for the constants $a$ and $b$, so that the value of $Y_{c}$ may be computed for any desired value of $X$. The values of a and $\mathrm{b}$ must be calculated in some way from the observed values of $\mathrm{X}$ and $\mathrm{Y}$ the relationship between which the line is intended to show.

FIRST CONDITION TO BE SATISFIED

In order that a regression line may be considered a good fit, it must first of all satisfy the condition that the sum of the values read off the line corresponding to the different $\mathrm{X}$-values should equal the sum of the observed Y-values, i.e. that

$$
\Sigma Y_{c}=\Sigma Y
$$

where $\mathrm{Y}$ is an observed $\mathrm{Y}$-value, and $\mathrm{Y}_{\mathrm{c}}$ a value read off the line. This condition may alternatively be stated in the following form. If $\mathrm{d}$ denotes the deviation of a point from the regression line:

$$
\begin{aligned}
\mathrm{d} & =\mathrm{Y}-\mathrm{Y}_{\mathrm{c}} \\
\Sigma \mathrm{d} & =\Sigma \mathrm{Y}-\Sigma \mathrm{Y}_{\mathrm{a}}
\end{aligned}
$$

whence if the condition defined by (2) holds:

$$
\Sigma \mathrm{d}=0
$$

which is an alternative way of stating the first condition. This means that

-F. C. Millo-Statistical Methods. Henry Holt \& Co., New York. 
the sum of the deviations of the points above the line must exactly balance the deviations of those below it.

It will now be shown that if $\Sigma \mathrm{d}=0$, the regression line must pass through the point $\overline{\mathrm{X}}, \overrightarrow{\mathrm{Y}}$; and that this will be true no matter what is the slope of the regression line. The point $\vec{X}, \vec{Y}$ is the point that would be secured by plotting the mean value of $\mathrm{Y}$ over the mean value of $\mathrm{X}$. A numerical example will illustrate what is meant. Table 1 gives a comparison between $a$ set of $\mathrm{Y}$-values and $\mathrm{Y}_{\mathrm{e}^{\text {-values }}}$ computed from the equation $Y_{c}=34-3 X$. This equation represents a line that would pass through the point $\bar{X} \bar{Y}$, for when $X=6$ (the mean of the $X$-values), $Y_{c}=34-(3 \times 6)$ $=16$, which is the mean of the Y-values.

TABLE 1

\begin{tabular}{|c|c|c|c|c|c|}
\hline $\mathbf{X}$ & $\mathrm{Y}$ & a & $b X$ & $Y_{c}$ & d \\
\hline 1 & 3 & +34 & -3 & 31 & -28 \\
\hline 3 & 11 & $"$ & -9 & 25 & $-14 \overrightarrow{\mathrm{X}}=-=6$ \\
\hline 5 & 14 & $"$ & -15 & 19 & -5 \\
\hline 8 & 15 & $"$ & -24 & 10 & +5 \\
\hline 9 & 20 & $"$ & -27 & 7 & $+13 \bar{Y}=-=16$ \\
\hline 10 & 33 & $"$ & -30 & 4 & +29 \\
\hline 36 & 96 & & & 96 & 0 \\
\hline$=\Sigma X$ & $=\Sigma Y$ & & & $=\Sigma Y_{c}$ & $=\Sigma d$ \\
\hline
\end{tabular}

$\Sigma X=$ sum of the $\mathrm{X}$-values, $\mathrm{\Sigma Y}=$ sum of the observed $\mathrm{Y}$-values.

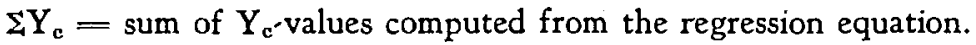

$\mathrm{d}=\mathrm{Y}-\mathrm{Y}_{\mathrm{c}}$, or the deviation of each $\mathrm{Y}$-value from the regression line. $\Sigma \mathrm{d}=$ sum of deviations.

$\overline{\mathrm{X}}=$ mean of the $\mathrm{X}$-values, $\overline{\mathrm{Y}}=$ mean of the $\mathrm{Y}$-values.

Although the trend of the $Y_{c}$-values does not at all correspond to the trend of the $Y$-values, their sum is the same. This is because the negative deviations of the first three $\mathrm{Y}$-values exactly balance the positive deviations of the last three.

The following algebraic proof of the above proposition may be given. Let it be assumed that a line be drawn blindly without necessarily passing through the point $\bar{X}, \bar{Y}$. Unless it be exactly vertical, it must crass the ordinate

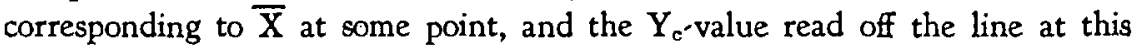
point may be designated $Y_{c}^{\prime}$. As may be seen from Fig. 1, a $Y_{c}$-value read 
off the line at an ordinate corresponding to any $\mathrm{X}$-value, may be defined by the equation

$$
\mathrm{Y}_{\mathrm{c}}=\mathrm{Y}_{\mathrm{c}}{ }+\mathrm{b}(\mathrm{X}-\overline{\mathrm{X}})
$$

The deviation of any point from the line will then be

$$
\begin{aligned}
\mathrm{d} & =\mathrm{Y}-\mathrm{Y}_{\mathrm{c}} \\
& =\mathrm{Y}-\mathrm{Y}_{\mathrm{c}}-\mathrm{b}(\mathrm{X}-\overline{\mathrm{X}}) \\
\mathrm{\Sigma} \mathrm{d} & =\mathrm{\Sigma}_{\mathrm{Y}} \mathrm{N}-\mathrm{NY^{ \prime }}{ }_{\mathrm{c}}-\mathrm{b} \Sigma_{\mathrm{C}}(\mathrm{X}-\overline{\mathrm{X}}) \\
& =\Sigma \mathrm{Y}-\mathrm{NY^{ \prime }}-\mathrm{b}(\Sigma \mathrm{X}-\mathrm{N} \overline{\mathrm{X}})
\end{aligned}
$$

where $\mathrm{N}$ is the total number of observations.

But since by the definition of an arithmetic average,

$$
\begin{aligned}
\bar{X} & =\frac{\Sigma X}{N} \\
N \bar{X} & =\Sigma X \\
b(\Sigma X-N X) & =0
\end{aligned}
$$

and therefore

$$
\Sigma \mathrm{d}=\mathbf{\Sigma} \mathrm{Y}-\mathrm{NY}_{\mathrm{c}} \mathrm{c}
$$

This will be true no matter what the position of the line but if the condition is to be satisfied that

$$
\Sigma d=0
$$

then it must follow that

$$
\begin{aligned}
N Y^{\prime}{ }_{\mathrm{o}} \text { must } & =\Sigma \mathrm{Y} \\
\mathrm{Y}_{\mathrm{c}}^{\prime} \text { must } & =\frac{\Sigma \mathrm{Y}}{\mathrm{N}}=\overline{\mathrm{Y}}
\end{aligned}
$$

and

In other words in order that the first condition that the regression line must satisfy will hold, the $Y_{c}$-value corresponding to the mean value of $X$, must be equal to the mean value of $Y$, i.e. the line must pass through the point $\overrightarrow{\mathrm{X}}, \overrightarrow{\mathrm{Y}}$.

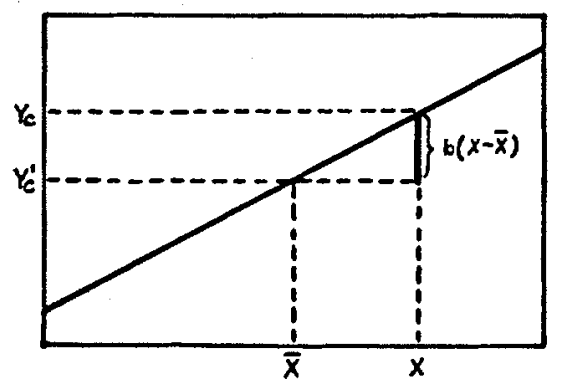

Fig. 1-Relation between $Y_{c}$ and $Y_{c}^{\prime}$.

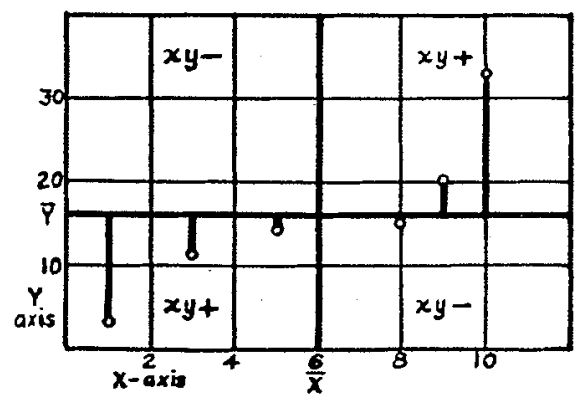

Fig. 2-Moments about horizontal line corresponding to mean value of $Y$. 


\section{SECOND CONDITION TO BE SATISFIED}

It may be noted above that the term including $\mathrm{b}$ disappears, which is the algebraic proof that the satisfying of the first condition does not depend at all on the slope of the line. It remains therefore to determine what second condition must be satisfied in order that the line may have the correct slope, or in other words that the coefficient $b$ in the equation of the line may have the correct value.

Since any line passing through the point $\vec{X}, \vec{Y}$, will satisfy the first condition, we may consider as a preliminary the situation which exists with respect to one of these possible lines, namely the horizontal line shown in Fig. 2, which corresponds to the mean value of $Y$. The points plotted in this figure correspond to the $\mathrm{X}$. and $\mathrm{Y}$-values of Table 1 . This horizontal line may be considered as a trial line which may be shifted to a different position in order to have it better represent the general relationship between the $X$. and $\mathrm{Y}$-values. But since it must in every case pass through the point $\overline{\mathrm{X}}, \overline{\mathrm{Y}}$, it can only be shifted by rotating it around this point. Obviously in order to have it fit the plotted points best, it should pass as closely as possible to them. Each point may be looked upon as exerting a force on the line, tending to pull it towards itself, the direction of the force being perpendicular along an ordinate, and its magnitude proportional to the distance of the point from the line. The farther the point is from the line, the greater the force exerted. The magnitudes of the forces exerted by the various points are indicated in Fig. 2 by heavy vertical lines. It is obvious however that of two points equally distant from the line, that which is further removed from the pivotal point $\overline{\mathrm{X}}, \overline{\mathrm{Y}}$ will exert the greater influence and that in general the distance of each point from the pivotal point must be taken into con. sideration. This may be done by taking as the effect or moment of the force exerted by each point, the product of its vertical distance from the horizontal line and its horizontal distance from the ordinate passing through the point $\overline{\mathrm{X}}, \overline{\mathrm{Y}}$. This is analogous to the method of computing the moments of forces in physics. The quantities entering into the computation of the moment of the force exerted by each point, as described above will be respectively $\mathrm{Y}-\overline{\mathrm{Y}}=\mathrm{y}$, and $\mathrm{X}-\overline{\mathrm{X}}=\mathrm{x}$, and their product will be xy. When $Y>\bar{Y}$, y will be positive, and when $Y<\bar{Y}$, y will be negative and the same with $x$. The products $x y$ will be positive when $x$ and $y$ have the same sign and negative when they have different signs. The horizontal and vertical lines through the point $\bar{X}, \vec{Y}$, divide the area of the graph into four quadrants and points located in two of these will have positive xy's and in the other two negative xy's as indicated in Fig. 2. 'It may be seen that points having positive xy's will tend to shift the line in a clockwise direction 
and those having negative xy's in a counter-clockwise direction. Whether the algebraic sum of all the xy's is positive or negative will indicate whether in general the line should be shifted in a clockwise or a counter-clockwise direction, that is whether it should slope up or down.

The numerical values of $\mathrm{xy}$ for the data of Table 1 are computed in Table 2.

TABLE 2

\begin{tabular}{rrrrrc}
\hline $\mathrm{X}$ & $\mathrm{Y}$ & $\mathrm{x}$ & $\mathrm{y}$ & $\mathrm{xy}$ & $\mathrm{x}^{2}$ \\
\hline 1 & 3 & -5 & -13 & +65 & +25 \\
3 & 11 & -3 & -5 & +15 & +9 \\
5 & 14 & -1 & -2 & +2 & +1 \\
8 & 15 & +2 & -1 & -2 & +4 \\
9 & 20 & +3 & +4 & +12 & +9 \\
10 & 33 & +4 & +17 & +68 & +16 \\
\hline & & & & +160 & +64 \\
& & & & $=\Sigma \mathrm{xy}$ & $=\Sigma \mathrm{x}^{2}$ \\
\hline
\end{tabular}

(The values of $\mathrm{x}^{2}$ are given here for later reference.)

The line may now be pictured as being shifted to a position such as that shown in Fig. 3 where it passes through the center of the band of points. The points still exert forces tending to shift the line but it is obvious that the forces tend to balance one another. The points representing the first and last pairs of $\mathrm{X}$ - and $\mathrm{Y}$-values tend to pull the line in a clockwise and the other four points in a counter-clockwise direction.

The magnitude of each force will be $Y-Y_{c}=d$, where $d$ is the deviation of each point from the line, measured vertically as before. These forces are represented in Fig. 3 by heavy lines, the same as in Fig. 2. The moments of the forces exerted by the points will be the products $\mathrm{dx}$. The line will be in equilibrium with respect to these forces when the positive dx's equal the negative $\mathrm{dx}$ 's, that is when

$$
\Sigma \mathrm{dx}=0
$$

This then is the second condition that the line must satisfy and it is finally necessary only to find how to determine what the slope of the line must be to satisfy this condition. This may be done by expressing $d$ in terms of $x$ and $y$, the values of which may be determined from the data.

By definition,

$$
\begin{aligned}
\mathrm{d} & =\mathrm{Y}-\mathrm{Y}_{\mathrm{c}} \\
& =(\mathrm{Y}-\overline{\mathrm{Y}})-\left(\mathrm{Y}_{0}-\overline{\mathrm{Y}}\right) \\
& =\mathrm{y}-\mathrm{bx}
\end{aligned}
$$


These relationships are illustrated in Fig. 4.

It follows that

$$
\begin{aligned}
d x & =x y-b x^{2} \\
\Sigma d x & =\Sigma x y-b \Sigma x^{2}
\end{aligned}
$$

and since $\Sigma \mathrm{d} \mathbf{x}$ must $=0$

$$
\Sigma x y-b \Sigma x^{2} \text { must }=0
$$

whence

$$
b=\frac{\Sigma x y}{x^{2}}
$$

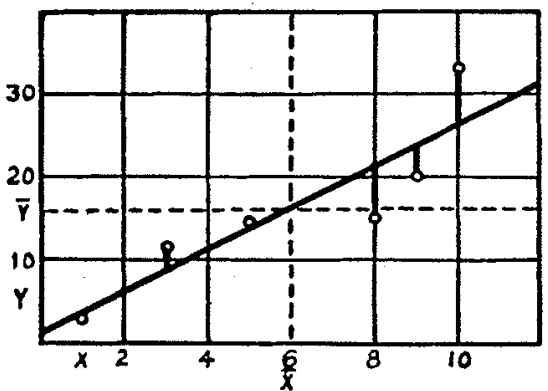

FIG. 3-Moments about regression line.

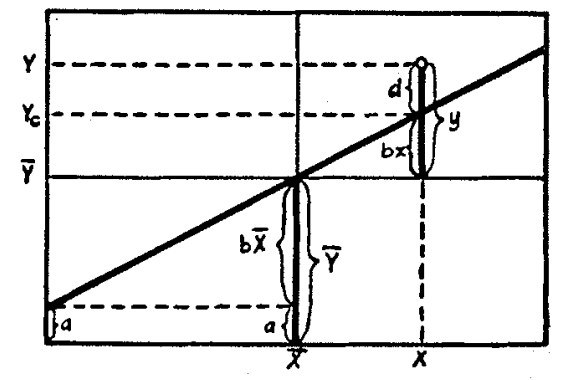

FIG. 4.

Since this equation is derived from the condition, $\Sigma \mathrm{d} x=0$, it defines $\mathrm{b}$ in such a way as to ensure that that condition will be satisfied.

Referring again to Fig. 4, it may be seen that

$$
\mathrm{a}=\overline{\mathrm{Y}}-\mathrm{b} \overline{\mathrm{X}}
$$

which is the most convenient way of computing the value of $a$ in order to complete the equation of the line

$$
Y_{c}=a+b X
$$

Algebraically, equation (6) is derived as follows:

$$
\begin{aligned}
Y_{c} & =a+b X \\
\Sigma Y_{c} & =\Sigma Y=N a+b \Sigma X \\
\frac{\Sigma Y}{N} & =a+\frac{b \Sigma X}{N} \\
a & =\bar{Y}-b \bar{X}
\end{aligned}
$$


The numerical values of $\Sigma x y$ and $\Sigma x^{2}$ are computed in Table 2, so that

$$
\begin{aligned}
b & =\frac{\Sigma x y}{\Sigma x^{2}} \\
& =\frac{+160}{64} \\
& =+2.50 \\
a & =16-2.50 \times 6 \\
& =1.00
\end{aligned}
$$

The equation of the regression line of best fit is therefore

\begin{tabular}{|c|c|c|c|c|c|c|c|}
\hline $\mathrm{X}$ & $\mathrm{Y}$ & $a$ & $b x$ & $Y_{c}$ & $d$ & $x$ & $\mathrm{dx}$ \\
\hline 1 & 3 & 1.0 & 2.5 & 3.5 & -0.5 & -5 & +2.5 \\
\hline 3 & 11 & $"$ & 7.5 & 8.5 & +2.5 & -3 & -7.5 \\
\hline 5 & 14 & ". & 12.5 & 13.5 & +0.5 & -1 & -0.5 \\
\hline 8 & 15 & $"$ & 20.0 & 21.0 & -6.0 & +2 & -12.0 \\
\hline 9 & 20 & " & 22.5 & 23.5 & -3.5 & +3 & -10.5 \\
\hline 10 & 33 & “ & 25.0 & 26.0 & +7.0 & +4 & +28.0 \\
\hline 36 & 96 & & & 96.0 & 0.0 & & 0.0 \\
\hline$=\Sigma X$ & $=\Sigma Y$ & & & $=\Sigma, Y_{c}$ & $=\Sigma \mathrm{d}$ & & $=\Sigma \mathrm{d} d x$ \\
\hline
\end{tabular}

$$
\mathrm{Y}_{\mathrm{c}}=1.0+2.5 \mathrm{X}
$$

Values computed from these equations and the resulting values of $d$ and $\mathrm{dx}$ are computed in Table 3.

TABLE 3

It will be seen that $\Sigma Y_{c}=\Sigma Y$ and $\Sigma d=0$ so that the first condition is satisfied, and $\Sigma \mathrm{dx}=0$ so that the second condition is also satisfied.

USE OF AN ARBITRARY ORIGIN

In actual computation work the mean values of $\mathrm{X}$ and $\mathrm{Y}$ seldom have integral values, and it would be very laborious computing the values of $\Sigma x y$ and $\Sigma x^{2}$ if two or three decimals entered into every value of $x$ and $y$. The values of $\Sigma x y$ and $\Sigma x^{2}$ may however be derived from $\Sigma X Y$ and $\Sigma X^{2}$ by means of the following reduction formulas:

$$
\begin{aligned}
& \Sigma x y=\Sigma X Y-\frac{\Sigma X \cdot \Sigma Y}{N} \\
& \Sigma x^{2}=\Sigma X^{2}-\frac{(\Sigma X)^{2}}{N}
\end{aligned}
$$


The values of the terms on the right side are computed from the observed values in their original form, or as it is sometimes stated, as deviations from the natural origin.

Since these values may be numerically rather large, it is more convenient to perform the necessary computations by taking deviations from integral values near the means of $\mathrm{X}$ and $\mathrm{Y}$, which are termed arbitrary origins. These arbitrary origins may be designated $O_{x}$ and $O_{y}$ and the deviations $x^{\prime}$ and $y^{\prime}$. The values of $\Sigma x y$ and $\Sigma x^{2}$ are then computed by the formulas:

$$
\begin{aligned}
& \Sigma x y=\Sigma x^{\prime} y^{\prime}-\frac{\Sigma x^{\prime} \Sigma y^{\prime}}{N} \\
& \Sigma x^{2}=\Sigma x^{\prime 2}-\frac{\left(\Sigma x^{\prime}\right)^{2}}{N}
\end{aligned}
$$

These formulas are identical in principal with (7) and (8) differing only in the substitution of $x^{\prime}$ and $y^{\prime}$ for $X$ and $Y$.

The derivation of formula (9) for computing $\Sigma_{x y}$ is as follows:

$$
\begin{aligned}
\mathrm{x} & =\mathrm{X}-\overline{\mathrm{X}} \\
& =\left(\mathrm{X}-\mathrm{O}_{\mathrm{x}}\right)-\left(\overline{\mathrm{X}}-\mathrm{O}_{\mathrm{x}}\right) \\
& =\mathrm{x}^{\prime}-\left(\overline{\mathrm{X}}-\mathrm{O}_{\mathrm{x}}\right)
\end{aligned}
$$

'But since by definition

$$
\begin{gathered}
X-O_{x}=x^{\prime} \\
\Sigma X-N O_{x}=\Sigma x^{\prime}
\end{gathered}
$$

Dividing by $\mathrm{N}$ :

$$
\begin{aligned}
& \frac{\Sigma X}{N}-O_{x}=\frac{\Sigma x^{\prime}}{N} \\
& ' \bar{X}^{\prime}-O_{x}=\frac{\Sigma x^{\prime}}{N}
\end{aligned}
$$

Substituting in (11):

$$
x=x^{\prime}-\frac{\Sigma x^{\prime}}{N}
$$


Similarly

$$
\begin{aligned}
y & =y^{\prime}-\frac{\Sigma y^{\prime}}{N} \\
x y & =\left(x^{\prime}-\frac{\Sigma x^{\prime}}{N}\right)\left(y^{\prime}-\frac{\Sigma y^{\prime}}{N}\right) \\
& =x^{\prime} y^{\prime}-x^{\prime} \cdot \frac{\Sigma y^{\prime}}{N}-y^{\prime} \cdot \frac{\Sigma x^{\prime}}{N}+\frac{\Sigma x^{\prime} \cdot \Sigma y^{\prime}}{N^{2}} \\
\Sigma x y & =\Sigma x^{\prime} y^{\prime}-\frac{\Sigma x^{\prime} \cdot \Sigma y^{\prime}}{N}-\frac{\Sigma y^{\prime} \cdot \Sigma x^{\prime}}{N}+N \frac{\left(\Sigma x^{\prime} \cdot \Sigma y^{\prime}\right)}{N^{2}} \\
& =\Sigma x^{\prime} y^{\prime}-\frac{\Sigma x^{\prime} \cdot \Sigma y^{\prime}}{N}-\frac{\Sigma y^{\prime} \cdot \Sigma x^{\prime}}{N}+\frac{\left(\Sigma x^{\prime} \cdot \Sigma y^{\prime}\right)}{N}
\end{aligned}
$$

The last three terms are identical, but the last two being opposite in sign cancel one another, leaving

$$
\Sigma x y=\Sigma x^{\prime} y^{\prime}-\frac{\Sigma x^{\prime} \cdot \Sigma y^{\prime}}{N}
$$

Formula (10) for computing $\Sigma x^{2}$ is similarily derived: Starting with formula (12) above,

$$
\begin{aligned}
x & =x^{\prime}-\frac{\Sigma x^{\prime}}{N} \\
x^{2} & =x^{\prime 2}-2 x^{\prime} \cdot \frac{\Sigma x^{\prime}}{N}+\left(\frac{\Sigma x^{\prime}}{N}\right)^{2} \\
\Sigma x^{2} & =\Sigma x^{\prime 2}-\frac{2 \Sigma x^{\prime} \cdot \Sigma x^{\prime}}{N}+\frac{N\left(\Sigma x^{\prime}\right)^{2}}{N^{2}} \\
& =\Sigma x^{\prime 2}-\frac{2\left(\Sigma x^{\prime}\right)^{2}}{N}+\frac{\left(\Sigma x^{\prime}\right)^{2}}{N} \\
& =\Sigma x^{\prime 2}-\frac{\left(\Sigma x^{\prime}\right)^{2}}{N}
\end{aligned}
$$

Table 4 gives a numerical example of the use of arbitrary origins. 
TABLE 4

\begin{tabular}{|c|c|c|c|c|c|c|c|}
\hline $\mathrm{X}$ & $\mathrm{Y}$ & $\mathrm{O}_{x}=4$ & $O_{x}=20$ & $x^{\prime} y^{\prime}$ & $x^{\prime 2}$ & $\mathrm{~s}$ & $x^{\prime} s$ \\
\hline 1 & 3 & -3 & -17 & +51 & 9 & -20 & +60 \\
\hline 3 & 11 & -1 & -9 & +9 & 1 & -10 & +10 \\
\hline 5 & 14 & +1 & -6 & -6 & 1 & -5 & -5 \\
\hline 8 & 15 & +4 & -5 & -20 & 16 & -1 & -4 \\
\hline 9 & 20 & +5 & 0 & 0 & 25 & +5 & +25 \\
\hline 10 & 33 & +6 & +13 & +78 & 36 & +19 & +114 \\
\hline $\begin{aligned} & 36 \\
&= \Sigma X \\
&\end{aligned}$ & $\begin{array}{r}96 \\
-\Sigma Y \\
\end{array}$ & $\begin{array}{l}+12 \\
=\Sigma x^{\prime}\end{array}$ & $\begin{array}{l}-24 \\
=\Sigma y^{\prime}\end{array}$ & $\begin{array}{l}+112 \\
=\Sigma x^{\prime} y^{\prime}\end{array}$ & $\begin{aligned} & 88 \\
&= \Sigma x^{\prime 2} \\
&\end{aligned}$ & $\begin{array}{l}-12 \\
=\Sigma \mathrm{s}\end{array}$ & $\begin{array}{l}+200 \\
=\Sigma \mathrm{x}^{\prime} \mathrm{s}\end{array}$ \\
\hline \multicolumn{8}{|c|}{$\left(\right.$ Note $\left.-s=x^{\prime}+y^{\prime}\right)$} \\
\hline \multicolumn{4}{|c|}{$\Sigma x y=\Sigma x^{\prime} y^{\prime}-\frac{\Sigma x^{\prime} \cdot \Sigma y^{\prime}}{N}$} & \multicolumn{4}{|c|}{$\Sigma x^{2}=\Sigma x^{\prime 2}-\frac{\left(\Sigma x^{\prime}\right)^{2}}{N}$} \\
\hline \multicolumn{5}{|c|}{$(+12) \times(-24)$} & \multicolumn{2}{|c|}{$=88-12^{2}$} & \\
\hline & \multicolumn{4}{|c|}{$=+112-(-48)$} & \multicolumn{3}{|c|}{$=88-24$} \\
\hline
\end{tabular}

In the computation of $\Sigma x^{2}$, it does not matter whether $\Sigma x^{\prime}$ is positive or negative, since its square will always be positive. In the case of $\Sigma x y$, the signs of $\Sigma x^{\prime}$ and $\Sigma y^{\prime}$ must be carefully watched.

Further checks on the computation work are illustrated in Table 4, the checks being summarized by the following formulas which may be applied to the numerical results in the table:

$$
\begin{aligned}
& \Sigma X=\Sigma x^{\prime}+N O_{x} \\
& \Sigma Y=\Sigma y^{\prime}+N O_{y} \\
& \Sigma_{s}=\Sigma x^{\prime}+\Sigma_{y^{\prime}} \\
& \Sigma_{i} x^{\prime} s=\Sigma x^{\prime 2}+\Sigma x^{\prime} y^{\prime}
\end{aligned}
$$

By the use of arbitrary origins, the maximum magnitude of the values of $x^{\prime}$ and $y^{\prime}$ may be kept to about one-half of the maximum magnitudes of the $X^{\prime} s$ and $Y^{\prime} s$, and the values of $x^{\prime} y^{\prime}$ and $x^{\prime 2}$ to about one-quarter of their ratural values.

In connection with formulas (9) and (10), a careful distinction should be made between the meaning of $\Sigma x^{\prime}$. $\Sigma y^{\prime}$ and $\Sigma x^{\prime} y^{\prime}$, and also between $\left(\Sigma x^{\prime}\right)^{2}$ and $\Sigma x^{\prime 2}$. The notation $\Sigma x^{\prime}$ represents simply the total of a column of figures, in which each entry is the value of an individual $x^{\prime}$. The notation $\Sigma x^{\prime} . \Sigma y^{\prime}$ represents the product of that total and the total of a similar column 
of $y^{\prime}$ - values. The notation $\Sigma x^{\prime} y^{\prime}$ on the other hand represents the total of a column in which the entries are each the product of an individual pair of $x^{\prime}-$ and $y^{\prime}-$ values. Similarly the notation $\Sigma x^{\prime 2}$ represents the total of a column in which the entries are each the square of an individual $x^{\prime}-$-value.

\section{METHOD OF NORMAL EQUATIONS}

The simultaneous solution of two normal equations is more usually given as the method of determining the constants of a linear regression equation. These normal equations take the form:

$$
\begin{aligned}
\Sigma Y & =\mathrm{Na}+\mathrm{b} \Sigma X \\
\Sigma X Y & =\mathrm{a} \Sigma \mathrm{X}+\mathrm{b} \Sigma \mathrm{X}^{2}
\end{aligned}
$$

or using an arbitrary origin:

$$
\begin{aligned}
\Sigma y^{\prime} & =N a+b \Sigma x^{\prime} \\
\Sigma x^{\prime} y^{\prime} & =a \Sigma x^{\prime}+b \Sigma x^{\prime 2}
\end{aligned}
$$

They are derived by the calculus from the general condition

$$
\Sigma \mathrm{d}^{2}=\mathrm{a} \text { minimum }
$$

from which the "Method of Least Squares" derives its name. The two conditions $\Sigma \mathrm{d}=0$ and $\Sigma \mathrm{dx}=\mathrm{O}$ may similarly be derived from this single general condition.

The method outlined above however appears to be more convenient than the solution of normal equations in the case of linear regression, although the latter is much preferable where there are more than two constants in the regression equation.

\section{SUMMARY}

It has been shown that a linear regression equation should satisfy two conditions.

$$
\begin{aligned}
\Sigma d & =0 \\
\Sigma d x & =0
\end{aligned}
$$

and the meaning of these conditions has been fully explained.

From these conditions the following formulas for $a$ and $b$ in the regression equation have been derived:

$$
\begin{aligned}
& b=\frac{\Sigma x y}{\Sigma x^{2}} \\
& a=-\vec{Y}-b \bar{X}
\end{aligned}
$$

Various details of notation and methods of computation have been men. tioned. The main object has been to show as clearly as possible the relations that exist between a linear regression line and the data to which it is fitted, and why the algebraic method of calculating the equation of the line secures the desired results. 